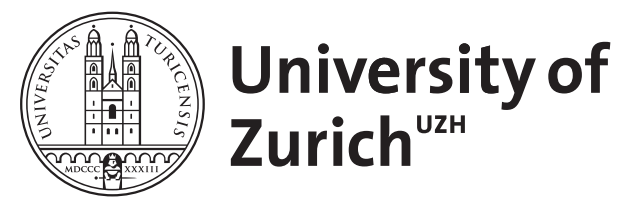

\title{
Vibration and rotation in millimeter-wave SAR
}

Rüegg, M ; Meier, E ; Nüesch, D

\begin{abstract}
Synthetic aperture radar (SAR) provides high-resolution images of static ground scenes, whereas processing of data containing ground object motion results in varying focusing effects. Special cases of such motion are vibration and rotation, which are closely related to each other. Their patterns may be distinctly recognizable in focused SAR intensity images as well as in a time-frequency analysis. Millimeter-wave ( $\mathrm{mmW}$ ) SAR is well suited to image vibration because its wavelength is close to typical vibration amplitudes. Through a thorough motion analysis in a standard SAR system model, we show the effects of rotation and vibration in mmW SAR theoretically and in simulated and real data.
\end{abstract}

DOI: https://doi.org/10.1109/TGRS.2006.887025

Posted at the Zurich Open Repository and Archive, University of Zurich ZORA URL: https://doi.org/10.5167/uzh-62918

Journal Article

Published Version

Originally published at:

Rüegg, M; Meier, E; Nüesch, D (2007). Vibration and rotation in millimeter-wave SAR. IEEE Transactions on Geoscience and Remote Sensing, 45(2):293-304.

DOI: https://doi.org/10.1109/TGRS.2006.887025 


\title{
Vibration and Rotation in Millimeter-Wave SAR
}

\author{
Maurice Rüegg, Student Member, IEEE, Erich Meier, and Daniel Nüesch, Member, IEEE
}

\begin{abstract}
Synthetic aperture radar (SAR) provides highresolution images of static ground scenes, whereas processing of data containing ground object motion results in varying focusing effects. Special cases of such motion are vibration and rotation, which are closely related to each other. Their patterns may be distinctly recognizable in focused SAR intensity images as well as in a time-frequency analysis. Millimeter-wave $(\mathrm{mmW}) \mathrm{SAR}$ is well suited to image vibration because its wavelength is close to typical vibration amplitudes. Through a thorough motion analysis in a standard SAR system model, we show the effects of rotation and vibration in mmW SAR theoretically and in simulated and real data.
\end{abstract}

Index Terms-Micro-Doppler, millimeter-wave (mmW) radar, synthetic aperture radar (SAR), time-frequency analysis.

\section{INTRODUCTION}

$\mathbf{F}$ OR a long time, moving targets in synthetic aperture radar (SAR) and ground moving target indication (GMTI) algorithms for SAR have intrigued large parts of the radar community [1]. Apart from constant target motion, less common movements like rotation have been an issue ever since [2], and micro-Doppler analysis of vibration, rotation, and related motion patterns still is [3]-[5]. SAR imaging of moving targets is discussed in [6, Ch. 7] and micro-Doppler phenomena of vibration and rotation in [6, Ch. 8]. Similar SAR micro-Doppler analyses are presented in [7].

As implied by the name micro-Doppler, vibration induces very small Doppler frequency modulations in a radar signal. They originate from a target oscillation of at most tens of hertz with amplitudes being no more than a few millimeters. Clearly, millimeter-wave $(\mathrm{mmW})$ radar systems are especially well suited to detect such small oscillations. Vibration amplitudes affect $\mathrm{mmW}$ radar wavelengths much more than longer carrier wavelengths. Non-SAR studies of vibration analysis in $\mathrm{mmW}$ radar have recently been conducted [8]. In this paper, we show the effects of vibration in SAR as well as why rotation is a special case of vibration. Whereas in [3], micro-Doppler theory is discussed in detail, we lay our main focus on an accurately described system and simulation model as well as on real data from plausible targets, namely results of vehicle vibrations and radar dish rotations.

Manuscript received April 21, 2006; revised July 17, 2006. This work was supported by the Procurement and Technology Center, Swiss Federal Department of Defence (armasuisse).

M. Rüegg and E. Meier are with the Remote Sensing Laboratories, University of Zurich, 8057 Zurich, Switzerland (e-mail: mrueegg@geo.unizh.ch).

D. Nüesch, retired, was with the Remote Sensing Laboratories, University of Zurich, 8057 Zurich, Switzerland.

Digital Object Identifier 10.1109/TGRS.2006.887025

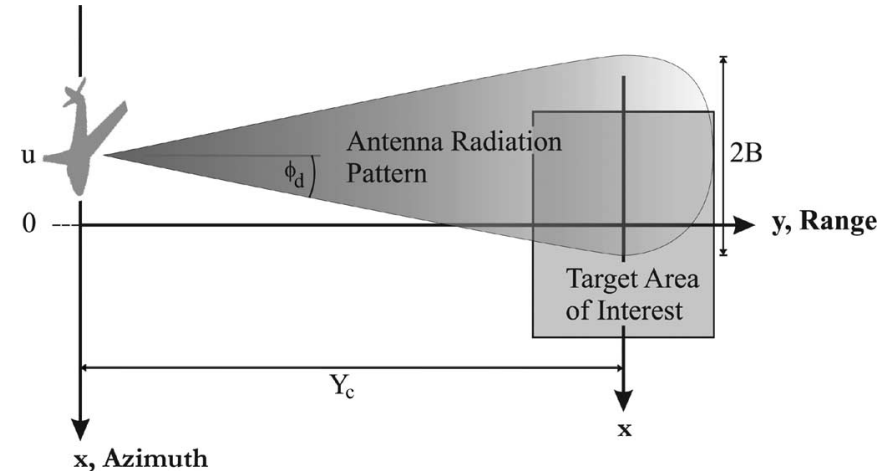

Fig. 1. Two-dimensional SAR geometry for the system model used.

It is not the aim of this paper to present SAR GMTI algorithms [9]-[12]; instead, we focus on only two mathematically describable object movements, namely: 1) vibration and 2) rotation, and their effects on SAR images. To be able to do this, we first define a simple 2-D radar geometry and a SAR system model for static point targets, which were used as a basis for simulations in Section II. We extend this model in Section III to include vibrating and rotating point targets in SAR. Additionally, we take a closer look at the micro-Doppler effect and discuss analysis possibilities with time-frequency distributions [13]. We analyze simulated data in Section IV and real scenes imaged with the Defense Research Facility for Applied Science (FGAN) multi-frequency experimental monopulse high-resolution interferometric SAR (MEMPHIS) mmW SAR at 94-GHz carrier frequency in Section V [14]. We conclude our observations in Section VI.

\section{SYSTEM MOdel}

The following SAR geometry and system model for static point targets are based on [15]. Because we use them as a foundation to all subsequent considerations, they are explained in some detail in this paper.

\section{A. Geometry}

To simplify the analysis without loss of generality, we use a purely 2-D sensor flight geometry, with the $x$-coordinate pointing down in the flight direction (azimuth) and the $y$-coordinate pointing right in the radar look direction (range). We assume an airborne stripmap SAR system on a linear flight track and neglect spaceborne data effects of Earth rotation and orbit curvature. All image dimensions are given by the target area of interest, the SAR antenna radiation pattern, and the resulting recorded scene, as described in Fig. 1. 
The antenna radiation pattern defines the 3-dB beamwidth of SAR. We model the signal inside this $3-\mathrm{dB}$ beamwidth through its transfer function, which is given as

$$
\begin{aligned}
& H(\omega, x-u, y)=a(\omega,x-u, y) \\
& \cdot \exp \left(-2 j \frac{\omega}{c} \sqrt{(x-u)^{2}+y^{2}}\right) .
\end{aligned}
$$

Obviously, the phase $\exp (\cdot)$ as well as the amplitude $a(\cdot)$ depends on the instantaneous signal frequency $\omega$ and the relative position between a scatterer at $(x, y)$ and the SAR sensor at $(u, 0) . c$ is the speed of light.

The area of validity (3- $\mathrm{dB}$ beamwidth) of the antenna is strongly dependent on the type of antenna installed (either planar array or curved antenna) and its specifications. For our model, we use a simple pattern defined via the half-beamwidth $B$ as follows:

$$
B(y)=y \cdot \tan \left(\phi_{d}\right)
$$

This implies the knowledge of the size of the antenna aperture angle $\phi_{d}$, which is approximated in [15] via the wavelength $\lambda_{c}$ of the carrier frequency and the physical antenna diameter in azimuth $D_{x}$ as

$$
\phi_{d}=\operatorname{asin}\left(\frac{\lambda_{c}}{D_{x}}\right) .
$$

Finally, let $Y_{c}$ be the range to the center of the area of interest.

\section{B. SAR Signal Model}

After having defined a target geometry, we would like to define a model for the raw data as received by the radar for analysis and simulation. Raw data have the advantage over single look complex focused SAR images in that we hold an additional level of information, namely the unprocessed amplitude and phase of targets useful for a time-frequency analysis.

We start by defining the emitted radar signal. Many SAR systems use a frequency-modulated linear chirp signal. Thus, we define such a chirp $p$ as a function of the fast time $t$ (i.e., the time in range $y$ defined via $c$ ) for our system model as

$$
p(t)=e^{j\left(\beta t+\alpha t^{2}\right)} .
$$

Here, $\alpha$ is known as the chirp rate, and $\beta$ is a linear coefficient. Equation (4) implies that we use a carrier frequency $\omega_{c}=\beta+$ $\alpha \tau_{p}$, where $\tau_{p}$ is the pulse duration, and the signal bandwidth is $\pm \omega_{0}= \pm \alpha \tau_{p}$. The Fourier transform of (4) is defined as

$$
P(\omega)=\int_{-\infty}^{\infty} p(t) \cdot e^{-j \omega t} d t
$$

Together with the transfer function from (1) and using the stopand-go approximation (assuming no sensor movement between signal transmission and reception, which is a reasonable assumption for airborne $\mathrm{SAR})$, we receive an echo $s(t, u)$ at the antenna position $u$ from all point targets $n$ at locations $\mathbf{Q}_{n}=\left(x_{n}, y_{n}\right)$ given by its Fourier transform

$$
\begin{aligned}
S(\omega, u)= & P(\omega) \cdot \sum_{n} A_{n} \cdot H_{n}\left(\omega, x_{n}-u, y_{n}\right) \\
= & P(\omega) \cdot \sum_{n} A_{n} \cdot a_{n}\left(\omega, x_{n}-u, y_{n}\right) \\
& \cdot \exp \left(-2 j \frac{\omega}{c} \sqrt{\left(x_{n}-u\right)^{2}+y_{n}^{2}}\right) .
\end{aligned}
$$

Whereas the term $A_{n}$ is the physical target reflectivity, $a_{n}$ is the return echo amplitude of a point target $n$ with unit reflectivity. More exactly, we use the target aspect angle

$$
\theta_{n}(u)=\operatorname{atan}\left(\frac{x_{n}-u}{y_{n}}\right), \quad-\phi_{d} \leq \theta_{n}(u) \leq \phi_{d}
$$

to define the signal amplitude of the $n$th target as a raised cosine

$$
a_{n}\left(\omega, x_{n}-u, y_{n}\right)=\frac{1}{2}+\frac{1}{2} \cos \left(\frac{\pi \theta_{n}(u)}{\phi_{d}(\omega)}\right) .
$$

We note that the antenna aperture angle is given depending on $\omega$, which may be important for wideband systems. Instead of (3), we may set $\phi_{d}(\omega)=\operatorname{asin}\left(2 \pi c / \omega D_{x}\right)$. With $u \in$ $\left[x_{n}-B_{n}, x_{n}+B_{n}\right]$ and $B_{n}=y_{n} \tan \left(\phi_{d}\right)$, we receive a target amplitude different from zero within the synthetic aperture of the radar.

Finally, we shift our received raw SAR signal to the baseband with

$$
s_{B}(t, u)=s(t, u) \cdot e^{-j \omega_{c} t} .
$$

\section{THEORETICAL ANALYSIS}

The system model presented in Section II offers the opportunity to define target motion quite easily by declaring the target position to be a function of the slow time or sensor azimuth time

$$
T=u / v_{s}
$$

where $v_{s}$ is the sensor speed. We define the position $\mathbf{Q}_{n}(T)$ of target $n$ at slow time $T$ to be

$$
\mathbf{Q}_{n}(T)=\left(\begin{array}{l}
x_{n}(T) \\
y_{n}(T)
\end{array}\right)
$$

It is well known that a moving target may be smeared, defocused, and displaced in azimuth in a focused SAR image [1]. This displacement is due to a Doppler shift that depends on a radial target velocity and is defined as

$$
f_{D_{n}}(T)=\frac{\omega_{c} v_{r_{n}}(T)}{\pi c}
$$

with the instantaneous radial target speed given by

$$
v_{r_{n}}(T)=\frac{d \mathbf{Q}_{n}(T)}{d T} \cdot \frac{\mathbf{r}_{n}(T)}{\left|\mathbf{r}_{n}(T)\right|}
$$


where $\mathbf{r}_{n}(T)$ is the radial look vector. If we introduce instantaneous target velocities $v_{x_{n}}(T)$ in $x$ and $v_{y_{n}}(T)$ in $y$, we get

$$
v_{r_{n}}(T)=v_{x_{n}}(T) \sin \left(\theta_{n}(T)\right)+v_{y_{n}}(T) \cos \left(\theta_{n}(T)\right)
$$

using the definition of $\theta_{n}(u)$ in (7) and the relation between $u$ and $T$ in (10). The Doppler shift of (12) is superimposed on the basebanded radar signal as

$$
s_{B_{n}}(t, T)=s_{n}(t, T) \cdot e^{-j\left(\omega_{c}+2 \pi f_{D_{n}}(T)\right) t}
$$

where $s_{n}(t, T)$ is the echo signal of target $n$. The amount of target displacement in azimuth is given as [1], [16]

$$
d_{n}(T)=-\frac{v_{r_{n}}(T)}{v_{s}}\left|\mathbf{r}_{n}(T)\right| .
$$

Because of the target movement and change of variables from azimuth position $u$ to slow time $T$, the target aspect angle $\theta_{n}(u)$ defined in (7) must be adapted to

$$
\theta_{n}(T)=\operatorname{atan}\left(\frac{x_{n}-T v_{s}+T v_{x_{n}}}{y_{n}+T v_{y_{n}}}\right) .
$$

In Section III-A, we concentrate on small displacement effects and accompanying micro-Doppler effects, where the aspect angle plays a minor role while the Doppler frequency plays a major one.

\section{A. Micro-Doppler Effects}

Mechanical vibrations or rotations of radar targets induce phase modulations in the received signal. These modulations are caused by what is known as the micro-Doppler phenomenon, and they create sidebands in the Doppler frequency spectrum of the target [6].

1) Vibration: A vibration may originate from a wide range of objects-from a standing vehicle with a running engine (e.g., in a traffic jam) to large Earth surface areas shaking during an earthquake. As we will see, vibrations cause a superimposed phase modulation on the received SAR signals.

We assume effects to be dependent on sensor azimuth time $T$ and consider vibrations in range only because vibrations in azimuth result in insignificant smearing of objects (see [1]). Apart from the vibration, the target does not move. Hence, we define a point target $n$ by its vibration in range

$$
V_{n}(T)=A_{V_{n}} \cos \left(\omega_{V_{n}} T\right)
$$

with vibration amplitude $A_{V_{n}}$ and frequency $\omega_{V_{n}}$. The target's instantaneous position is

$$
\mathbf{Q}_{n}(T) \approx\left(\begin{array}{c}
x_{n} \\
y_{n}+V_{n}(T)
\end{array}\right) .
$$

The approximation is valid if we assume a large sensor to target distance and a small antenna aperture $\left(\left(x_{n}-u\right)^{2}+y_{n}^{2} \approx y_{n}^{2}\right)$ as is the case for mmW SAR. The target velocity components are $v_{x_{n}}=0$ and

$$
v_{y_{n}}(T)=\frac{d V_{n}}{d T}=-A_{V_{n}} \omega_{V_{n}} \sin \left(\omega_{V_{n}} T\right) .
$$

To calculate the target Doppler shift due to the vibration, we could use (12), (14), and (15). Equivalently, we may use (18) and the two-way change of phase due to vibration to get

$$
\Delta \varphi_{n}(T)=\frac{4 \pi}{\lambda_{c}} A_{V_{n}} \cos \left(\omega_{V_{n}} T\right)
$$

with the wavelength $\lambda_{c}=2 \pi c / \omega_{c}$. The vibration-induced micro-Doppler shift is

$$
\begin{aligned}
f_{D_{n}, \mathrm{vib}}(T) & =\frac{1}{2 \pi} \frac{d \Delta \varphi_{n}(T)}{d T} \\
& =\frac{-2 A_{V_{n}} \omega_{V_{n}}}{\lambda_{c}} \sin \left(\omega_{V_{n}} T\right) .
\end{aligned}
$$

We see that a vibrating target in SAR causes a phase modulation in the received signal phase of (15). We also see that this effect depends on the wavelength and is strongest for small $\lambda_{c} \mathrm{~S}$ as in mmW SAR.

The phase modulation causes an effect in SAR images known as paired echoes. As the name suggests, we get ghost targets above and below a vibrating target due to the Doppler shifts. As shown in [3] and [6], target vibration induces an infinite series of paired echoes $\kappa$ because, when considering (22), the received signal of (15) may be expressed by a series expansion with the Bessel function of the first kind of order $\kappa$, i.e.,

$$
J_{\kappa}(z)=\frac{1}{2 \pi} \int_{-\pi}^{\pi} e^{j(z \sin \nu-\kappa \nu)} d \nu
$$

where $z=A_{V_{n}}\left(4 \pi / \lambda_{c}\right)$, and $\nu=\omega_{V_{n}} T$. Since

$$
\lim _{\kappa \rightarrow \infty} J_{\kappa}(z)=0
$$

the paired echo signal strength decreases with increasing $\kappa$. As shown in (16), the displacement of the $\kappa$ th paired echo coming from the vibration of target $n$ is

$$
d_{n, \kappa}=\mp \frac{\kappa \lambda_{c} \omega_{V_{n}}}{4 \pi} \cdot \frac{1}{v_{s}} y_{n} .
$$

One last point to mention is that if the SAR carrier platform is an airplane, additional micro-Doppler frequencies may come from platform vibration. This is discussed in [3] and is closely related to target vibration.

2) Rotation: Examples of objects with rotating motion include ground-based surveillance radar antennas or helicopter rotors. They may have very different backscattering properties, but they show common movement and, therefore, similar Doppler characteristics. As presented in the following analysis, the Doppler signature from rotating motion is closely related to vibration.

We develop a model for an extended object consisting of multiple aligned point targets moving in a circle around a common center. All point targets $m$ of a rotating object $n$ share a common rotation frequency $\omega_{R_{n}}$ but have individual radii 


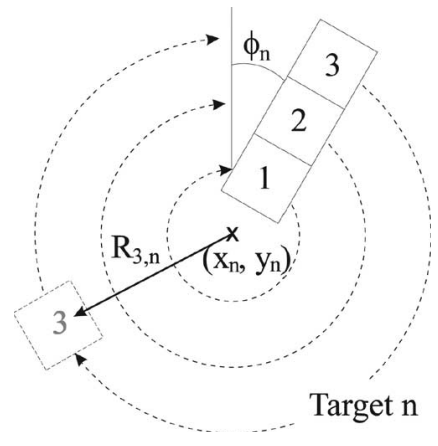

Fig. 2. Rotating target $n$ consisting of $m=3$ point targets with radii $R_{m, n}$ sharing a common center, rotation frequency, and phase offset $\phi_{n}$.

$R_{m, n}$ from the center $\left(x_{n}, y_{n}\right)$ of the object. We define the instantaneous position of such a point target as

$$
\mathbf{Q}_{m, n}(T)=\left(\begin{array}{l}
x_{n}+R_{m, n} \cos \left(\omega_{R_{n}} T+\phi_{n}\right) \\
y_{n}+R_{m, n} \sin \left(\omega_{R_{n}} T+\phi_{n}\right)
\end{array}\right)
$$

where $\phi_{n}$ is the initial angle of the target at time zero. Fig. 2 illustrates the idea behind (26).

The positions $\mathbf{Q}_{m, n}(T)$ may easily be incorporated into our standard model of (6) for the received echoes at the SAR antenna. Depending on the method, we either calculate the instantaneous radial velocity and the Doppler shift according to (12) or define the two-way phase change $\Delta \varphi_{m, n}(u)$ of $m$ as for vibrations. To use the instantaneous velocity is the direct approach; thus, we need the velocity vector given by

$$
\frac{d \mathbf{Q}_{m, n}}{d T}=\left(\begin{array}{c}
-R_{m, n} \omega_{R_{n}} \sin \left(\omega_{R_{n}} T+\phi_{n}\right) \\
R_{m, n} \omega_{R_{n}} \cos \left(\omega_{R_{n}} T+\phi_{n}\right)
\end{array}\right) .
$$

Together with (12) and (14), we may then add a rotationinduced micro-Doppler shift into (15), given as

$$
\begin{aligned}
& f_{D_{n}, \text { rot }}(T)=\frac{\omega_{c}}{\pi c}[-\sin \left(\omega_{R_{n}} T+\phi_{n}\right) \cos \left(\theta_{n}(T)\right) \\
&\left.\quad+\cos \left(\omega_{R_{n}} T+\phi_{n}\right) \sin \left(\theta_{n}(T)\right)\right] \cdot R_{m, n} \omega_{R_{n}} \\
&=-\frac{\omega_{c}}{\pi c}\left[\sin \left(\omega_{R_{n}} T+\phi_{n}-\theta_{n}(T)\right)\right] R_{m, n} \omega_{R_{n}} .
\end{aligned}
$$

As for vibration, we see that the micro-Doppler effect of a rotation is larger with smaller wavelength $\lambda_{c}$ or higher carrier frequency $\omega_{c}$, making mmW SAR suitable for detection of such effects.

An important aspect of rotating objects is their change of reflectivity during rotation. If we assume a ground-based planar radar antenna as our rotating object, it is clear that the antenna broadside will have a much higher reflectivity than its side profile.

Because a rotation implies a constantly changing velocity at every point of an object with nonzero $R_{m, n}$, we get many effects for rotating targets caused by the Doppler shift of (12). We will see this clearly when we look at simulated and real data below.

\section{B. Time-Frequency Analysis of Micro-Doppler}

The Fourier transform is a common method used to analyze radar signal waveforms in the frequency domain. MicroDoppler signatures are included in the Fourier spectrum as deviations in the Doppler spectrum. However, with the Fourier transform, we lose all information of time. A joint time-frequency analysis is needed to extract time-varying information in the spectrum of a radar signal.

A good bilinear time-frequency transform that belongs to Cohen's class [13] is the Wigner-Ville distribution (WVD). As such, it is an energy distribution in contrast to linear time-frequency representations, which decompose the signal into elementary components (atomic distributions). The WVD of a signal $s(t)$ is defined as the Fourier transform of the timedependent autocorrelation function, i.e.,

$$
W(t, \omega)=\int_{-\infty}^{\infty} s\left(t+t^{\prime} / 2\right) s^{*}\left(t-t^{\prime} / 2\right) \cdot e^{-j \omega t^{\prime}} d t^{\prime} .
$$

This definition requires the knowledge of the quantity

$$
s\left(t+t^{\prime} / 2\right) s^{*}\left(t-t^{\prime} / 2\right), \quad-\infty \leq t^{\prime} \leq \infty
$$

which is not the case for a sampled radar signal. A window filter $h(t)$, such as a Hamming window in the time domain, solves this problem and results in the pseudo-WVD. To reduce the cross-term interference problem of the WVD, we add a smoothing function $g(t)$ (e.g., a second Hamming window) and obtain the smoothed pseudo-WVD [17]

$$
\begin{aligned}
S W(t, \omega)= & \int_{-\infty}^{\infty} h\left(t^{\prime}\right) \int_{-\infty}^{\infty} g(y-t) \\
& \cdot s\left(t+t^{\prime} / 2\right) s^{*}\left(t-t^{\prime} / 2\right) d x \cdot e^{-j \omega t^{\prime}} d t^{\prime} .
\end{aligned}
$$

This distribution is a compromise between joint time-frequency resolution and the level of interference terms; i.e., the more one smoothes in time and/or frequency, the poorer the resolution in time and/or frequency [17].

In SAR, the echoes of successively transmitted pulses show a phase shift varying with azimuth time $T$ as a function of the relative motion between the radar and each backscatterer. Each point on the ground reflects a Doppler shift that is proportional to the projection of the radar velocity $v_{s}$ along the line of sight by the angle $\theta(T)$ and is defined by

$$
f_{D}(T)=\frac{v_{s} \omega_{c} \sin (\theta(T))}{\pi c} .
$$

This is basically the same relation as the one given in (12); the difference is that here, we have the Doppler history of a static target, whereas in (12), we have a superimposed Doppler frequency from target movement. For small $\mathrm{mmW}$ antenna apertures $\theta(T), f_{D}(T)$ may be assumed to be linear. 


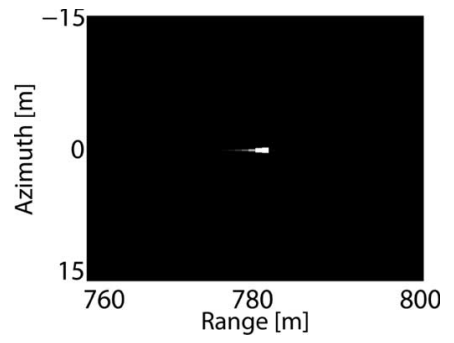

Fig. 3. Focused intensity image of a simulated static $94-\mathrm{GHz}$ SAR point target. Image resolution is $0.75 \mathrm{~m}$.

This Doppler history over time, as described in (32), can be made clearly visible with a time-frequency analysis. Additionally, Doppler shifts from moving targets as well as micro-Doppler phenomena may be observed in detail with the smoothed pseudo-WVD, as we will see when looking at simulated data in Section IV.

For simulated data, the smoothed pseudo-WVD is well suited. Real SAR data, however, are often corrupted by noise, and micro-Doppler effects may not be observed in either the time domain or the frequency domain. For this, atomic decompositions, such as the Gabor transform [13], [17], are very useful because, unlike an energy distribution like the WVD, they are localized in both the time and the frequency domains. Therefore, the Gabor transform is often used to visualize microDoppler effects [6], and we did additional analyses on all experimental targets with the Gabor transform. Since both representations showed congruent results, and to make a direct comparison between simulations and real data possible, we confined ourselves to presenting the ones from the smoothed pseudo-WVD in the Section IV.

\section{Analysis of Simulated Data}

With the presented system model and the theoretical analysis of micro-Doppler phenomena, we are able to simulate vibration and rotation in raw SAR data. A visual evaluation is then possible from focused images and from time-frequency analyses with smoothed pseudo-WVD. In the following, we present focused simulated mmW SAR images that have been processed with the extended chirp-scaling algorithm [18] as well as time-frequency analysis from pulse-compressed-only data. To start, we simulated a static point target in Fig. 3 .

For this intensity image, we used mmW SAR system parameters with a carrier frequency of $f_{c}=94 \mathrm{GHz}\left(\lambda_{c}=3.19 \mathrm{~mm}\right)$, a signal bandwidth of $2 \omega_{0}=200 \mathrm{MHz}$, a pulse duration of $\tau_{p}=1.2 \mu \mathrm{s}$, a pulse repetition frequency (PRF) of $1.7 \mathrm{kHz}$, and a SAR sensor speed in azimuth of $v_{s}=75 \mathrm{~m} / \mathrm{s}$. We chose an antenna with $D_{x}=0.12 \mathrm{~m}$. The target area of interest is located at $Y_{c}=780 \mathrm{~m}$. This results in a dwell time in which a point target is illuminated for approximately $0.5 \mathrm{~s}$. If not indicated otherwise, these same parameters are valid for all simulations and real data presented in this paper. In Fig. 4, the time-frequency representation of the pulse-compressed target's Doppler spectrum is given and confirms the SAR Doppler history of (32) for a static point target.

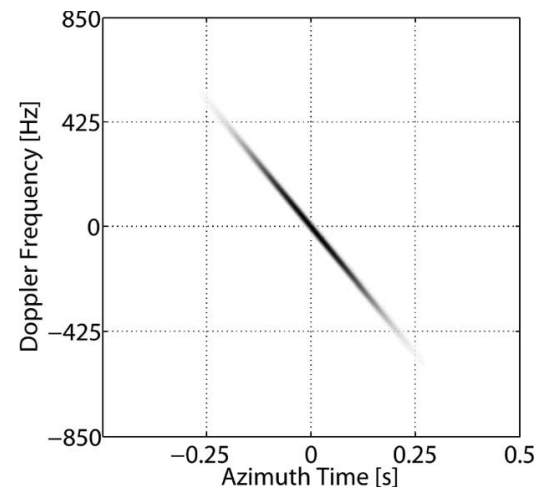

Fig. 4. Time-frequency representation of the pulse-compressed data from a simulated 94-GHz SAR point target.

\section{A. Vibration}

There is the possibility to analyze not only the Doppler spectrum of a target but also its signal spectrum. For a static point target and a chirped signal, this would be something very similar to the image in Fig. 4, but with a truly linear slope for a linear chirp instead of (32), a measurement time of only the pulse duration $(1.2 \mu \mathrm{s})$, and a much larger spectral bandwidth of megahertz instead of hertz. These are important points. If we want to analyze micro-Doppler effects with frequency values of at most tens of hertz, we are well advised to do so in the Doppler spectrum, where our measurement time-the dwell time of the radar-is much longer and frequency resolution is higher than in the signal spectrum.

In the focused SAR image of Fig. 5(a), we simulated five vibrating point targets, all with a vibration frequency of $30 \mathrm{~Hz}$. The vibration amplitude $A_{V_{n}}$ for targets from left to right is decreasing from 10 to $0.001 \mathrm{~mm}$. All vibrations are in range and perpendicular to the flight track, and zero movement in azimuth is assumed. Although targets with large vibration amplitudes clearly show multiple and very densely spaced paired echoes as theoretically predicted by (25), the paired echo signal strength of small vibration amplitudes is not visible at all. The spacing between paired echoes is a mere $0.08 \mathrm{~m}$.

To compare the influence of vibration frequency on target signatures, the simulation has been repeated with five targets vibrating at the same amplitudes but at a frequency of $50 \mathrm{~Hz}$, as shown in Fig. 5(b). As (25) predicts, the spacing between paired echoes increases and is equal to $0.13 \mathrm{~m}$. Once more, the amplitudes of 0.01 and $0.001 \mathrm{~mm}$ of the two targets to the right are too small for any visible effects.

Clearly, it is very difficult to measure target vibration frequencies from the displacement of paired echoes in focused SAR images. This is the case even for a large vibration amplitude of $1 \mathrm{~mm}$ at low frequencies of 30 and $50 \mathrm{~Hz}$, as the second targets from the left in Fig. 5(a) and (b) show. A time-frequency analysis gives much better information, as we may see in Fig. 6, where we analyzed four of the ten targets of Fig. 5 via a smoothed pseudo-WVD. Although the static Doppler slope is always visible in the data, we can clearly discern a vibration signature for all four targets and even approximate the vibration frequency by the naked eye when counting wave periods over time. In Fig. 6(a), we see a target vibrating at $30 \mathrm{~Hz}$ and with an 


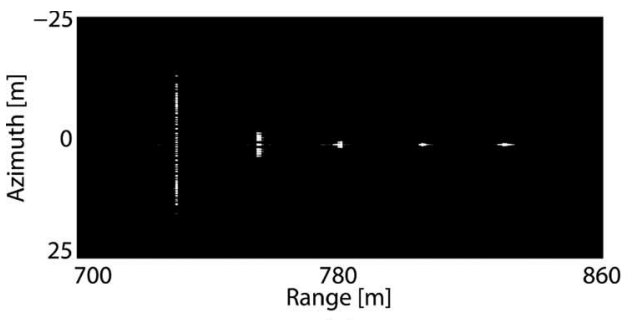

(a)

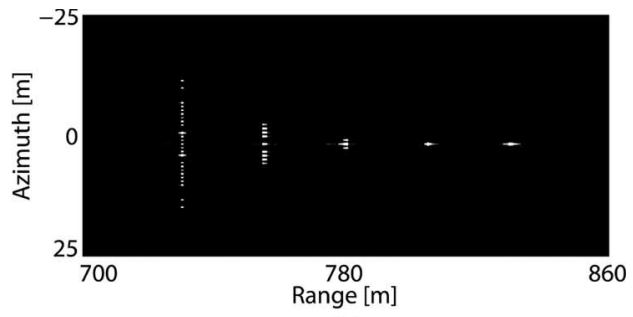

(b)

Fig. 5. Focused 94-GHz SAR images, each with five simulated targets vibrating at (a) $30 \mathrm{~Hz}$ and (b) $50 \mathrm{~Hz}$. Vibration amplitudes from left to right are 10 , $1,0.1$, 0.01 , and $0.001 \mathrm{~mm}$ for both images.

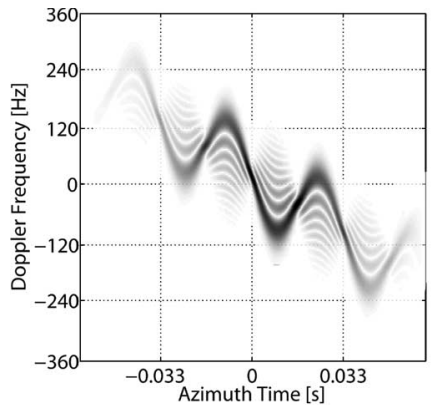

(a)

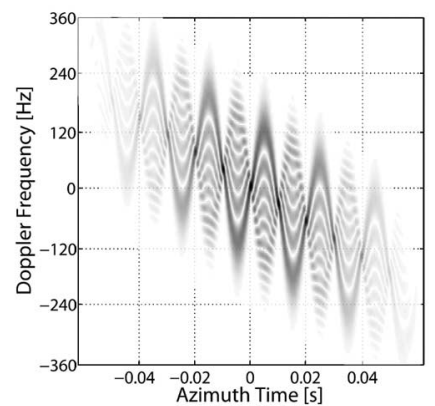

(c)

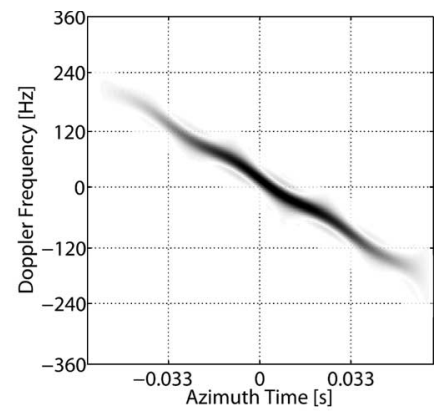

(b)

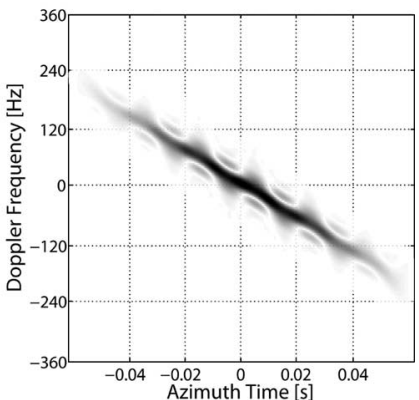

(d)

Fig. 6. Time-frequency representation (WVD) of four simulated SAR targets vibrating at frequencies of either 30 or $50 \mathrm{~Hz}$ and with amplitudes of $1.0 \mathrm{or}$ $0.1 \mathrm{~mm}$. (a) Vibration frequency of $30 \mathrm{~Hz}$ and amplitude of $1.0 \mathrm{~mm}$. (b) Vibration frequency of $30 \mathrm{~Hz}$ and amplitude of $0.1 \mathrm{~mm}$. (c) Vibration frequency of $50 \mathrm{~Hz}$ and amplitude of $1.0 \mathrm{~mm}$. (d) Vibration frequency of $50 \mathrm{~Hz}$ and amplitude of $0.1 \mathrm{~mm}$.

amplitude of $1.0 \mathrm{~mm}$. This means that the wave period must be $0.033 \mathrm{~s}$. The determination of the vibration amplitude is possible since (22) states that

$$
A_{V_{n}}=-\frac{f_{D_{n}, \operatorname{vib}}(T) \lambda_{c}}{2 \omega_{V_{n}}} .
$$

With $\omega_{V_{n}}=2 \pi \cdot 30 \mathrm{~Hz}$, an amplitude of $A_{V_{n}}=1.0 \mathrm{~mm}$ must result from a local maximum of $f_{D_{n}, \text { vib }}=118 \mathrm{~Hz}$, which may be extracted from the plot by removing the constant Doppler slope. In Fig. 6(b), the same vibration frequency and an amplitude of $0.1 \mathrm{~mm}$ results in a local maximum of $f_{D_{n} \text {,vib }}=$ $11.8 \mathrm{~Hz}$. We see that the interpretation of the target signature becomes harder. This agrees with our findings from the intensity image of Fig. 5(a). Still, we may discern a target vibration and approximate the vibration frequency. In the intensity image, this would be near impossible.

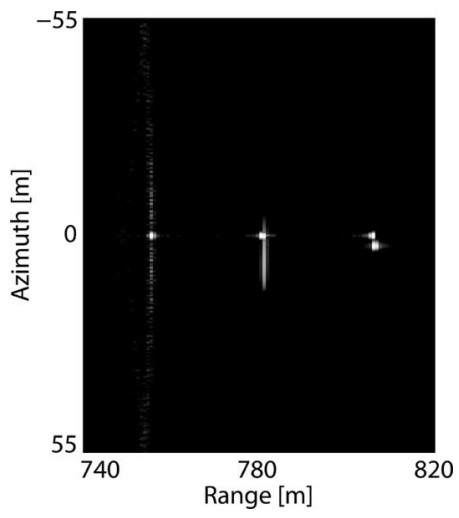

Fig. 7. Three simulated focused 94-GHz SAR targets rotating at (left) $10 \mathrm{~Hz}$, (center) $0.5 \mathrm{~Hz}$, and (right) $0.2 \mathrm{~Hz}$, all with a rotation radius of $0.5 \mathrm{~m}$.

For target vibrations of $50 \mathrm{~Hz}$, a time-frequency analysis in Fig. 6(c) and (d) shows corresponding results. Whereas the focused SAR image gives us almost no information on vibration, a smoothed pseudo-WVD identifies a wave period of $0.02 \mathrm{~s}$ and correct local maxima of $f_{D_{n}, \text { vib }}=118$ and $11.8 \mathrm{~Hz}$ after subtracting the constant Doppler slope.

\section{B. Rotation}

First results of rotating objects in SAR have been presented in [2]. We simulated the large variable displacement effects (smearing) that appear in an ideal situation (no background clutter) in Fig. 7, where three rotating objects are visible. We made the following assumptions as to the rotation movement: Each object is made up of two point targets, namely: 1) a static one, representing the immobile part of an object (and giving us a feeling of where the object is truly located in the SAR image) and 2) a rotating one, circling the static one at a distance of $R=0.5 \mathrm{~m}$. The reflectivity of the circling target does not change with aspect angle; this is an idealistic assumption, but it prevents the loss of information because of reflectivity changes that may occur during a rotation. To show that the smearing effects may be asymmetrical, we gave the rotation an offset of $\phi_{n}=\pi / 8$ for all targets as defined in (26). Finally, the rotation frequency was chosen to be $10 \mathrm{~Hz}$ for the left target, $0.5 \mathrm{~Hz}$ for the center target, and $0.2 \mathrm{~Hz}$ for the right target.

We may discern the individually sampled displacements of the target rotating with $10 \mathrm{~Hz}$ in Fig. 7 quite easily, and we may also identify range bin changes due to the radius of rotation. Obviously, the first phenomenon is heavily dependent on the SAR system PRF and the second one on target radius and SAR 


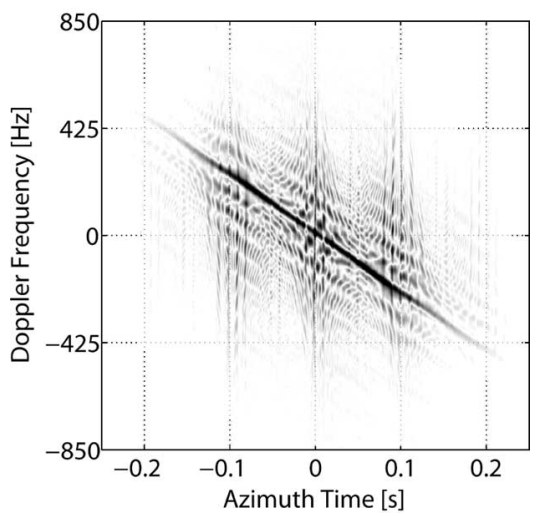

Fig. 8. Time-frequency representation (WVD) of a simulated target rotating at $10 \mathrm{~Hz}$. The pattern shows the relation to vibrations as given in Fig. 6.

resolution. The other two targets are harder to analyze because their rotation periods of 2 and $5 \mathrm{~s}$ are both larger than the SAR dwell time of $0.5 \mathrm{~s}$. We can only observe a partial period of rotation in the focused SAR image.

A time-frequency analysis shows good results that correspond well with theoretical results. In Fig. 8, the 10-Hz rotation is shown over a time interval of the full radar dwell time of $0.5 \mathrm{~s}$. We may easily identify the rotation period of $0.1 \mathrm{~s}$ per turn.

Additionally, a clear relation to target vibration may be seen in Fig. 8. If we were to fully ignore the small smearing effects caused by the rotation movement in azimuth, we would have a pure vibration at $10 \mathrm{~Hz}$ and an extremely large vibrational amplitude of $0.5 \mathrm{~m}$. Thus, the basic difference in real-world vibrations from real-world rotations is the magnitude of movement and frequency. This means that we may even use (33), which we stated for vibrations, to determine the radius of rotation from a time-frequency signature.

A much smaller rotation frequency of $0.5 \mathrm{~Hz}$, which is observed over the same time interval of $0.5 \mathrm{~s}$, shows the expected partial signal pattern in a time-frequency distribution, as given in Fig. 9(a) for the center target of Fig. 7. An approximation of the rotation frequency is possible since the time-frequency characteristic shows one-fourth of a sine wave in $0.5 \mathrm{~s}$. Similarly, the time-frequency representation for the target rotating with $0.2 \mathrm{~Hz}$ is shown in Fig. 9(b). A small deviation from the signature to be expected of a static target is visible, indicating a slow rotation. Unlike in Fig. 9(a), it becomes clear that such a small observation interval does not provide sufficient information on the movement in the time-frequency distribution. Such a slow rotation may best be extracted with traditional GMTI methods [12].

\section{ANALysis OF REAL DatA}

We conducted an experiment in the fall of 2005 with the airborne mmW SAR system MEMPHIS of the German Research Institute for High Frequency Physics and Radar Techniques (FGAN-FHR). A C-160 Transall, which is a large and stable aircraft, served as the radar platform. The system parameters of MEMPHIS match the ones from our simulations in Section IV with the addition that a depression angle of $20^{\circ}$ was used-the value is variable for MEMPHIS but dependent on the antenna

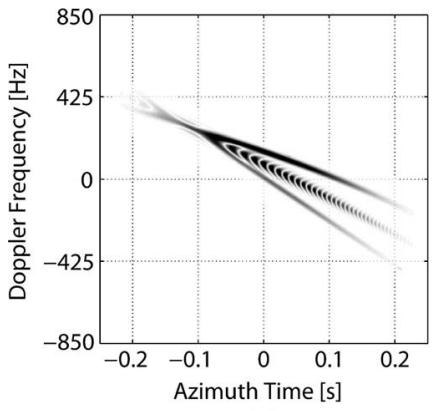

(a)

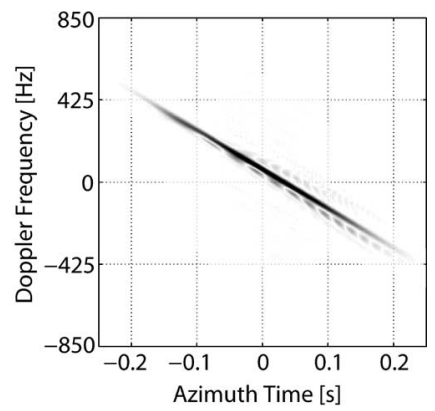

(b)
Fig. 9. Time-frequency representation (WVD) of two simulated rotating targets with individual rotation frequencies of (a) $0.5 \mathrm{~Hz}$ and (b) $0.2 \mathrm{~Hz}$, respectively.

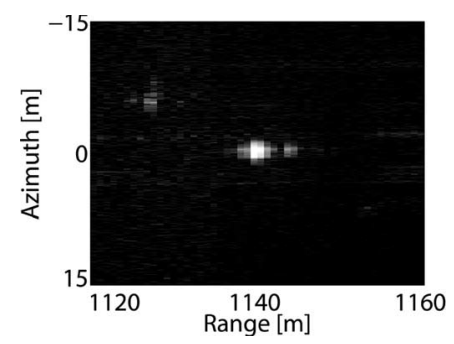

Fig. 10. Focused SAR image of a static corner reflector at the image center.

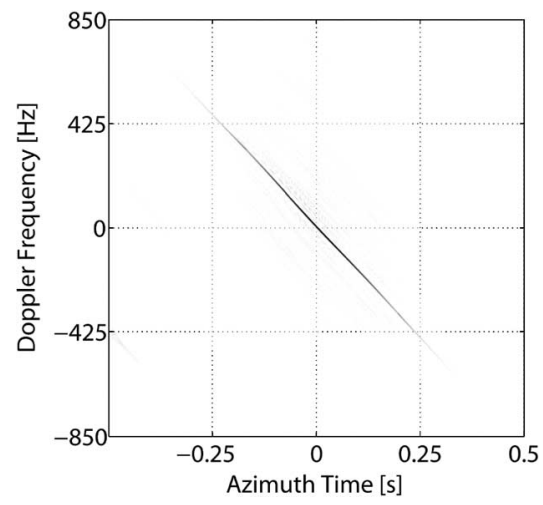

Fig. 11. Time-frequency representation of the pulse-compressed SAR data of the static corner reflector in Fig. 10.

characteristics for different range distances and sensor altitudes. The test site was an airfield tarmac and surrounding meadows in Switzerland. The following examples are all taken from this experiment.

As a first example and reference target, we analyzed a static corner reflector. The focused image of Fig. 10 shows this reflector at $94 \mathrm{GHz}$ and a resolution of $0.75 \mathrm{~m}$ at the center of the figure. The image was processed using the extended chirpscaling algorithm. Intensities are given in a logarithmic scale with a dynamic range of $65 \mathrm{~dB}$.

A time-frequency analysis with the smoothed pseudo-WVD is shown in Fig. 11. The Doppler spectrum appears centered in the image because we included a correction of the Doppler centroid in the analysis to make image interpretation easier and more comparable to simulation results, where we always had a Doppler centroid value of zero. The image shows ideal time-frequency characteristics as given in (32). 


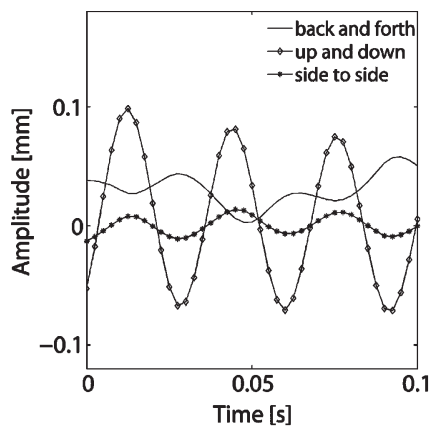

(a)

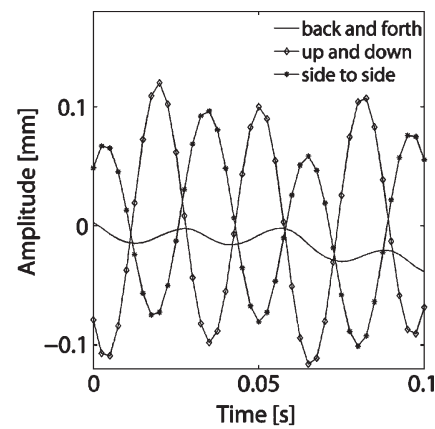

(b)
Fig. 12. Measured vibration amplitudes at the front bumper and on the cab roof of a truck running with an idle engine. (a) Front bumper. (b) Cab roof.

Fig. 11 indicates that we may ignore micro-Doppler effects from platform vibrations [3]. The distribution does not show any discernible micro-Doppler phenomenon, probably because MEMPHIS was carried on a very large and stable platform, the aforementioned $\mathrm{C}-160$. Hence, we assume platform motion to be negligible as well in the following MEMPHIS data.

\section{A. Vehicle Vibration}

Simulation results in Section IV show that whether or not target vibrations are visible may depend highly on vibration amplitude. Whereas higher vibration frequency causes paired echoes to be spaced farther apart from the actual target and more easily recognizable in a focused SAR image (see simulations in Fig. 5), it is higher amplitude that introduces clear vibration signatures of a target in the images as well as in a time-frequency representation. We are aware of but a single publication showing real data of vibration from a target other than a corner reflector in the open literature; in [19], data from a vibrating truck are given, but unfortunately, with no reference to the vibration parameters or SAR sensor. In [3] and [7], corner reflectors vibrating at $2 \mathrm{~Hz}$ and $1.5 \mathrm{~mm}$ amplitude in X-band SAR are presented, obviously with a very large amplitude and a low vibration frequency, making the targets easily detectable but less realistic.

Our own experiment with MEMPHIS included two identical modern-day trucks on the airfield tarmac. Prior to the experiment, truck vibration characteristics were measured by triaxial accelerometers, showing a linear frequency response from 0 to $150 \mathrm{~Hz}$ with an accuracy of $\pm 1 \%$ and an acceleration measurement range of $\pm 1 \mathrm{~g}$ with an accuracy of $\pm 1.5 \%$ [20]. Measurements were made for an idle engine and at higher rounds per minute. Because the largest vibrations were detected to be caused by an idle engine, we decided to use idle engine vibrations in our experiment. In Fig. 12(a) and (b), the idle motor engine vibration amplitudes are shown for a first measurement at the front bumper of a truck and for a second one on the cab roof. Maximum values are located at around $0.1 \mathrm{~mm}$ with strong variations of direction. Up and down movements on the truck roof were found to be the strongest.

In Fig. 13, the vibration frequency spectrum of such a truck with an idle engine is shown. Measurements were, again, done at the front bumper and on the roof. The peak frequency for

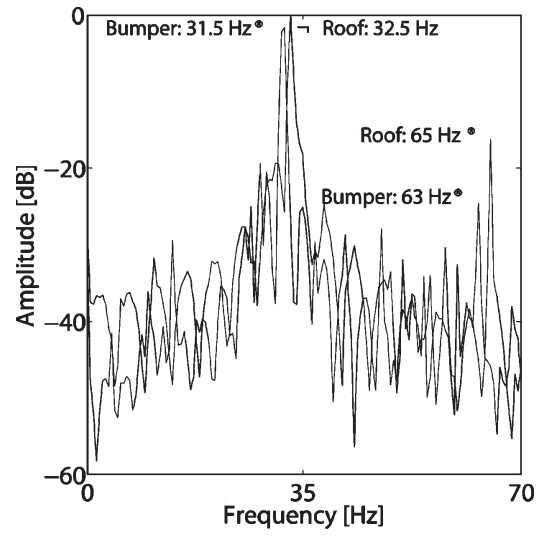

Fig. 13. Measured vibration frequencies at the front bumper and on the cab roof of a truck running with an idle engine.

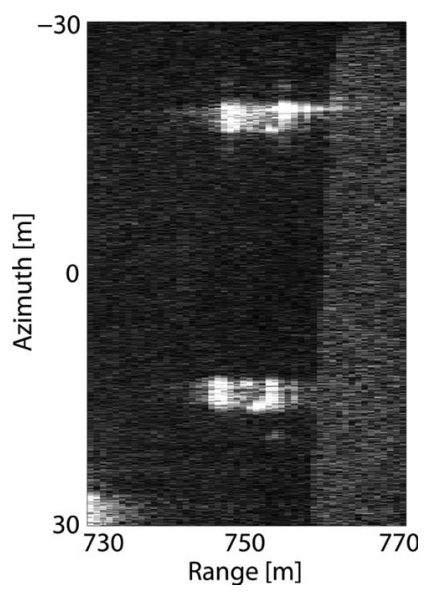

Fig. 14. Focused SAR intensity image of (top) a static and (bottom) a vibrating truck on a tarmac next to a meadow. No vibration-induced image signatures are recognizable.

both measurements was close to $32 \mathrm{~Hz}$ with a second harmonic at $64 \mathrm{~Hz}$, which is weaker than the peak by almost $20 \mathrm{~dB}$.

During the SAR experiment, the engine of one truck was turned off, and it was used as a permanent reference target during all data collection, whereas the second one had its engine running idly. They were both parallel to the range axis with their cabs facing the radar because we assumed the highest target returns to be coming from the metallic cab. The focused SAR image of the two trucks is given in Fig. 14. In the figure, no clear vibration-induced image signature is recognizable, and no paired echoes are visible. The target vibration amplitude was small, and the depression angle of SAR was not optimal at $20^{\circ}$, focusing on the weak back-and-forth movement instead of the somewhat stronger up-and-down movement.

In Fig. 15(a) and (b), the smoothed pseudo-WVD of the two trucks is given. We see that their time-frequency characteristics are very different from a corner reflector, which has to be expected when considering the many angles and faces of a truck, especially at mmW SAR. The plot for the static truck in Fig. 15(a) shows two very prominent areas at around $\pm 150 \mathrm{~Hz}$ Doppler. This may be caused by the space between the cab and the trailer, which acts as a corner reflector from certain angles. There is no clear pattern recognizable other than fluctuating variations of the signal intensity due to the truck geometry. 


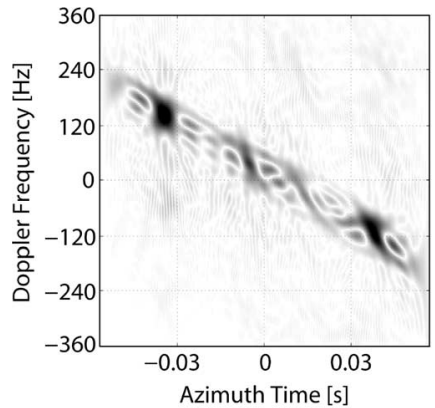

(a)

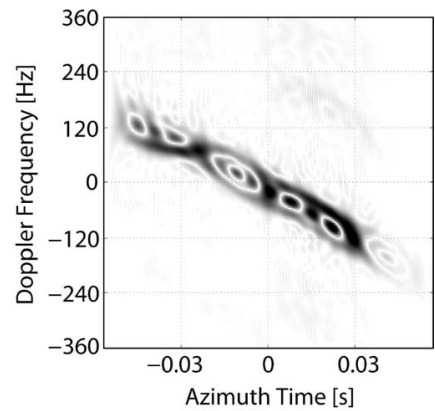

(b)
Fig. 15. Time-frequency representation (WVD) of the Doppler spectrum for (a) the static truck at the top and (b) the vibrating truck at the bottom of Fig. 14.

The plot for the vibrating truck in Fig. 15(b), however, appears to include a micro-Doppler pattern. We are able to discern a periodic signature at the confirmed vibration frequency of $32 \mathrm{~Hz}$ $(0.03 \mathrm{~s})$ and of its harmonics as well. Unfortunately, the prominent areas of high signal strength, which are well observable for the static truck, are located around the same frequencies as the ones that seem to be coming from vibration nodes. Because the signal becomes weak after about \pm 0.05 s due to the target geometry, it does not allow the detection of further vibration nodes. A larger vibration amplitude would certainly make an analysis easier and more confirmable. Vehicles with a larger amplitude, however, may be hard to find in the real world.

\section{B. Rotation of a Reflector and of a Radar Dish}

A second setup during the MEMPHIS data collection experiment described above included a rotating corner reflector turning with $1 \mathrm{~Hz}$ on a heavy wooden pedestal on the airfield tarmac. The reflector was a four-way trihedral reflector mounted to a lever arm that was moved by an electric motor, which allowed the rotation frequency to be tuned to $1 \mathrm{~Hz}$. The lever arm and the rotation radius were both $1 \mathrm{~m}$ long. The reflector was fixed to the lever arm and did not rotate on the arm. Hence, it did not provide a constant radar cross section. Its four-way reflection characteristics, however, prevented zero reflectivity from any angle and reduced the variation with a maximum cross section every $90^{\circ}$. Fig. 16 shows the pedestal, the lever arm, and the reflector during the experiment.

The rotating corner reflector was imaged with MEMPHIS during the same overflight as the two trucks. The resulting focused reflector is shown in Fig. 17 with a $0.75-\mathrm{m}$ resolution. At the top of the image, the pedestal is shown as a small target. The moving corner reflector is heavily smeared in azimuth, and because of low clutter returns from the tarmac, it is clearly visible. The signal from the rotation corner is asymmetrically smeared with no returns above the static pedestal because only a partial rotation was recorded by SAR. This is comparable to simulation results in Section IV (see Fig. 7). The dwell time of SAR over the target was approximately half a second.

To get reference data and offer change-detection possibilities, the corner reflector was also imaged when not moving during another overflight. These data confirmed an absent signal in regions whereto the corner reflector was smeared during rotation and a stronger signal at the pedestal and true corner location.

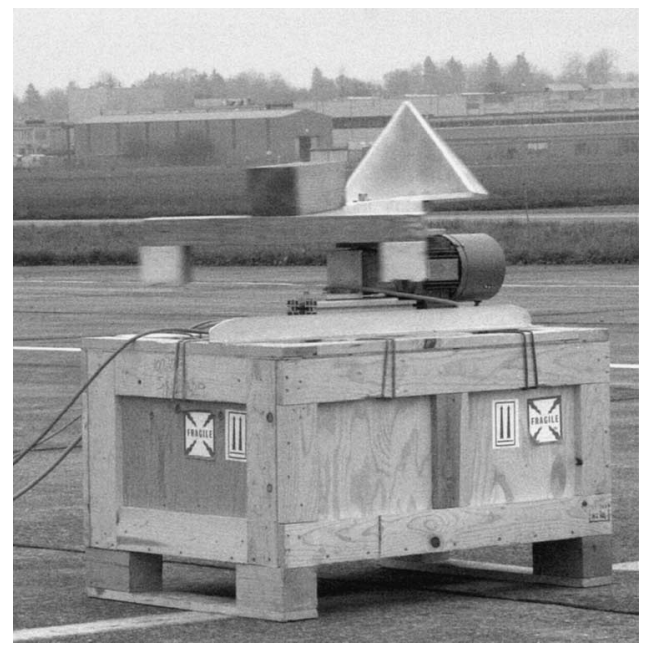

Fig. 16. Rotating corner reflector turning on a pedestal with $1 \mathrm{~Hz}$.

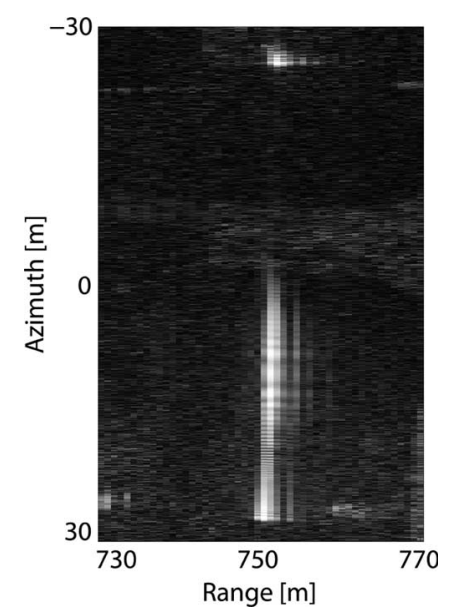

Fig. 17. Focused SAR intensity image of the rotating corner reflector in Fig. 16 turning on a pedestal with $1 \mathrm{~Hz}$. The pedestal is recognizable at the top of the image, whereas the rotating reflector is smeared in azimuth from center to bottom.

A time-frequency analysis with the smoothed pseudo-WVD of the rotating corner reflector in the pulse-compressed radar data gives us a very illustrative Doppler frequency spectrum over time, as shown in Fig. 18. Instead of a straight line as for a static target, we receive a curve clearly reflecting the varying acceleration of the corner during a rotation. Whereas the focused SAR intensity image in Fig. 17 may leave doubts about the origin of a smeared target signature, the time-frequency analysis proves the rotation movement of the reflector.

To show that the detection of rotation is not only possible for artificial targets, such as a rotating reflector, we also imaged the airfield air surveillance radar dish located nearby. Its antenna has a diameter of several meters and a rotation frequency of one complete turn every $4 \mathrm{~s}$. The impressive focused SAR intensity image is shown in Fig. 19. Whereas the asymmetrical experimental rotating corner reflector shows an asymmetrical SAR signature, the symmetrical surveillance radar dish gives a more point-symmetrical image. A signature with such large displacement effects for a small rotation frequency of $0.25 \mathrm{~Hz}$ and dwell time of $0.5 \mathrm{~s}$ is only possible if the timing between $\mathrm{SAR}$ and radar dish rotation is harmonized, which means a 


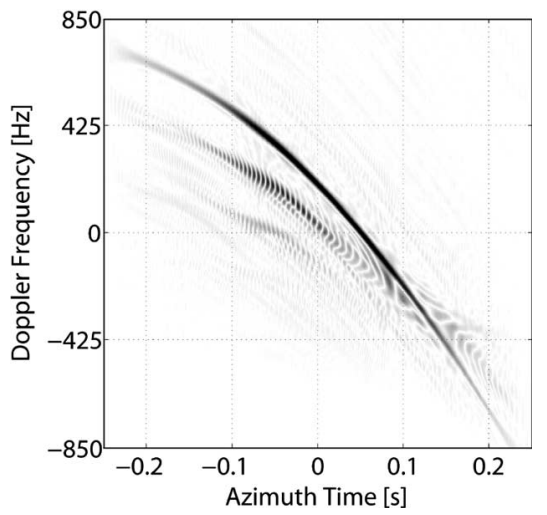

Fig. 18. Time-frequency representation of the corner reflector in Fig. 16, rotating at $1 \mathrm{~Hz}$

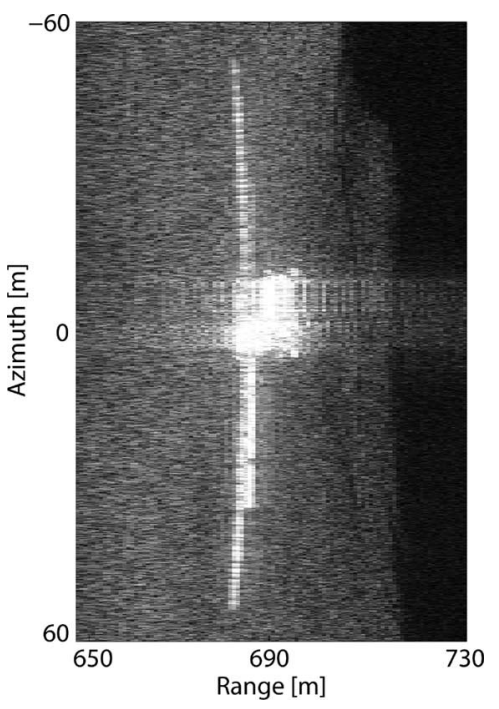

Fig. 19. Focused SAR intensity image of a ground-based radar antenna with a diameter of several meters, rotating at $0.25 \mathrm{~Hz}$.

frontal SAR imaging of the antenna dish. Imaging of the antenna dish from the side would mean a lower cross section in the SAR and radial velocities of close to zero, giving small displacement effects.

In Fig. 20, the time-frequency representation of the air surveillance radar dish is given. The signature shows a slight indication of a small fraction of a sine wave period, indicating a small rotation frequency of the target. After all, the radar dwell time of $0.5 \mathrm{~s}$ allows for only one-eighth of a rotation. Also shown are large returns of the static antenna pedestal. The advantage of the time-frequency representation is that, even if the imaging of the antenna had happened from the side with only small displacement effects in the SAR image, a curvature of the signature would eventually be visible.

If we are to estimate a rotation frequency of a target from its time-frequency analysis, we see a strong dependence on the dwell time. For the rotating corner reflector, we might be able to derive the actual rotation frequency of $1 \mathrm{~Hz}$ from the fact that half a sine wave is visible over $0.5 \mathrm{~s}$ in the time-frequency representation of Fig. 18. For the surveillance radar dish, an estimation of the rotation frequency is almost impossible because only a small fraction of a sine wave is

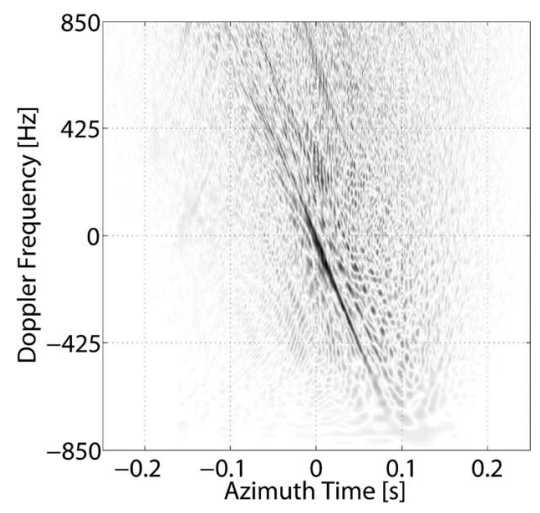

Fig. 20. Time-frequency representation of the ground-based radar antenna in Fig. 19.

visible in the time-frequency representation of Fig. 20. This is supported by the simulation results in Fig. 9.

\section{CONCLUSION}

We introduced the reader to the possibilities of airborne $\mathrm{mmW}$ SAR imaging of vibrating and rotating targets. The large range of potential applications may include anything from the detection of vehicles standing in traffic jams with idle engines to the reconnaissance of airfields, where rotating helicopter blades or ground-based radar dishes are present.

A system model that allows flexible simulations of vibration and rotation was proposed. It defines the instantaneous position of a target, its displacement effects in SAR imagery, and the Doppler shifts induced in the signal. An analysis of motion patterns in focused intensity images as well as in time-frequency distributions was conducted, showing microDoppler phenomena in simulations and in real data that match the underlying signal theory.

The time-frequency distributions presented to analyze vibration and rotation were done using the smoothed pseudoWVD, which is an energy distribution that is a compromise between joint time-frequency resolution and the level of interference terms. The Gabor transform, which is an atomic distribution that is well suited for data that are corrupted by noise and clutter, was considered as an alternative. Because the simulations were completely noise free and the real data included a low clutter and noise level, the presentation of only WVD results is justified.

From theoretical analyses, mmW SAR is well suited to detect vibration and rotation because it is very sensitive to micro-Doppler effects. Still, limitations are apparent for target vibrations with very small vibration amplitudes, such as the ones to be expected of a modern-day large truck with an engine running idly.

As seen in the simulated vibration data, a higher vibration frequency causes paired echoes to be spaced farther apart from the actual target and more easily recognizable in a focused SAR image, whereas a higher amplitude introduces clearer vibration signatures of a target in the images as well as in a time-frequency representation. Although we showed only a limited variation of vibration amplitude and frequency parameters, results hinted at well-detectable signal signatures for 
amplitudes larger than $0.1 \mathrm{~mm}$. Results for smaller amplitudes tend to be harder to interpret, as was confirmed by the real-data experiment with the two trucks.

The real data that we presented were recorded with MEMPHIS, which is an mmW SAR system. First, an analysis of a static corner reflector showed that aircraft vibration did not influence time-frequency analyses of real data. An evaluation of the focused SAR image of a static and a vibrating truck did not show any visible effects. This confirmed our simulation results that pointed at too closely spaced and too weakly paired echoes. The time-frequency distribution, however, did give an indication of vibration, although the interpretation of the pattern proved to be less distinct than hoped for after analyzing the simulations.

Regarding the time-frequency signature of a vibrating truck, it could be seen that target geometry may influence the return echoes to an extent where it becomes difficult to identify an underlying micro-Doppler phenomenon from such a weak vibration. The SAR sensor depression angle may be important when considering that up-and-down vibrations of a vehicle are much stronger than back-and-forth or side-to-side vibrations, as our ground-truth measurements of the trucks showed. An imaging from the side of the trucks may, in retrospect, have been useful, even though the main radar returns had to be expected from the metallic cab. A quantitative conclusion on vibration amplitude limitations, however, is difficult and may depend too heavily on assumptions in a simulation or on sensor and target characteristics for experimental data.

For rotating targets, difficulties arose when imaging slow rotational movements where the rotation period was much larger than the radar dwell time, which is generally very short for $\mathrm{mmW}$ SAR. Nevertheless, focused images of rotating targets showed clear target displacement into many azimuth bins along the target range. Time-frequency distributions clearly showed the micro-Doppler influence of rotation for simulated as well as for real data. Especially, the faster rotations of a corner reflector and an antenna dish proved to be useful for an estimate of rotation frequency. An increased dwell time of the radar could be a welcome measure to increase visibility of time-frequency characteristics of rotating targets.

Although vibration and rotation may be visible in a SAR intensity image, the origin of a smeared target signature or of paired echoes is most often not clear. A time-frequency analysis of such signatures, however, allows not only the target movement identification but also the measurement of vibration amplitude and frequency as well as rotation frequency and radius. An mmW sensor with a large dwell time and strong target vibrations of more than $0.1 \mathrm{~mm}$ or rotations where the rotation period is of the order of the dwell time seems to promise successful indication of both movements.

\section{ACKNOWLEDGMENT}

The authors would like to thank FGAN-FHR for the good cooperation, particularly H. Essen and M. Hägelen, as well as P. Wellig and K. Schmid of the Procurement and Technology Center, Swiss Federal Department of Defence (armasuisse), for their wide support and collaboration.

\section{REFERENCES}

[1] R. K. Raney, "Synthetic aperture imaging radar and moving targets," IEEE Trans. Aerosp. Electron. Syst., vol. AES-7, no. 3, pp. 499-505, May 1971.

[2] J. L. Walker, "Range-doppler imaging of rotating objects," IEEE Trans. Aerosp. Electron. Syst., vol. AES-16, no. 1, pp. 23-52, Jan. 1980.

[3] V. C. Chen, F. Li, S.-S. Ho, and H. Wechsler, "Micro-doppler effect in radar: Phenomenon, model, and simulation study," IEEE Trans. Aerosp. Electron. Syst., vol. 42, no. 1, pp. 2-21, Jan. 2006.

[4] S. D. Silverstein and C. E. Hawkins, III, "Synthetic aperture radar image signature of rotating objects," in Proc. Asilomar Conf. Signals, Syst., and Comput., Pacific Grove, CA, Nov. 2004, vol. 2, pp. 1663-1667.

[5] J. Li and H. Ling, "Application of adaptive Chirplet representation for ISAR feature extraction from targets with rotating parts," Proc. Inst. Electr. Eng.-Radar Sonar Navig., vol. 150, no. 4, pp. 284-291, Aug. 2003.

[6] V. C. Chen and H. Ling, Time-Frequency Transforms for Radar Imaging and Signal Analysis. Boston, MA: Artech House, 2002.

[7] T. Sparr and B. Krane, "Micro-doppler analysis of vibrating targets in SAR," Proc. Inst. Electr. Eng.-Radar Sonar Navig., vol. 150, no. 4, pp. 277-283, Aug. 2003

[8] D. Nüssler, H. Essen, and D. Büth, "Vehicle classification by vibration analysis using millimeterwave sensors," in Proc. Int. Conf. IrmmW, Williamsburg, VA, Sep. 2005, pp. 349-350.

[9] Applications of Space-Time Adaptive Processing, R. Klemm, Ed. London, U.K.: Institution Electrical Engineers, 2004.

[10] M. Soumekh, "Moving target detection and imaging using an X-band along-track monopulse SAR," IEEE Trans. Aerosp. Electron. Syst., vol. 38, no. 1, pp. 315-333, Jan. 2002.

[11] J. H. G. Ender, "Space-time processing for multichannel synthetic aperture radar," Electron. Commun. Eng. J., vol. 11, no. 1, pp. 29-38, Feb. 1999.

[12] M. Rüegg, M. Hägelen, E. Meier, and D. Nüesch, "Moving target indication with dual frequency millimeter wave SAR," in Proc. IEEE Conf. RADAR, Verona, NY, Apr. 2006, pp. 350-357.

[13] L. Cohen, "Time-frequency distributions-A review," Proc. IEEE, vol. 77, no. 7, pp. 941-981, Jul. 1989.

[14] H. Schimpf, H. Essen, S. Böhmsdorff, and T. Brehm, "MEMPHISA fully polarimetric experimental radar," in Proc. IGARSS, Toronto, ON, Canada, Jun. 2002, vol. 3, pp. 1714-1716.

[15] M. Soumekh, Synthetic Aperture Radar Signal Processing With MATLAB Algorithms. New York: Wiley, 1999, ch. 6. Stripmap Synthetic Aperture Radar, pp. 373-485.

[16] C. E. Livingstone, I. C. Sikaneta, C. H. Gierull, S. Chiu, A. Beaudoin, J. Campbell, J. Beaudoin, S. Gong, and T. A. Knight, "An airborne synthetic aperture radar (SAR) experiment to support RADARSAT-2 ground moving target indication (GMTI)," Can. J. Remote Sens., vol. 28, no. 6, pp. 794-813, Dec. 2002.

[17] F. Auger, P. Flandrin, P. Goncalves, O. Lemoine, Time-Frequency Toolbox for use With MATLAB, 1996. tutorial.pdf. [Online]. Available: http://tftb.nongnu.org/

[18] A. Moreira, J. Mittermayer, and R. Scheiber, "Extended chirp scaling algorithm for air-and spaceborne SAR data processing in stripmap and scanSAR imaging modes," IEEE Trans. Geosci. Remote Sens., vol. 34, no. 5, pp. 1123-1136, Sep. 1996.

[19] R. J. Sullivan, Microwave Radar: Imaging and Advanced Concepts. Boston, MA: Artech House, 2000.

[20] MS2002 + Acceleration Sensor, Syscom Instruments SA, Zurich, Switzerland, 2002.

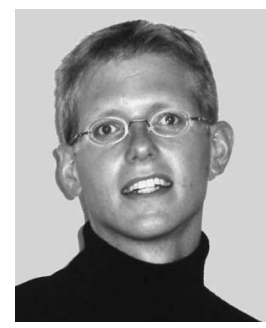

Maurice Rüegg (S'00) received the M.Sc. degree in electrical engineering from the Swiss Federal Institute of Technology (ETH), Zurich, Switzerland, in 2003. He is currently working toward the Ph.D. degree in microwave remote sensing at the Remote Sensing Laboratories, University of Zurich, Zurich, where he is analyzing the effects of and the detection techniques for ground moving targets in SAR and millimeter-wave radar data.

His research interests include imaging algorithms for various air- and spaceborne SAR sensors, SAR GMTI, and high-resolution SAR. 


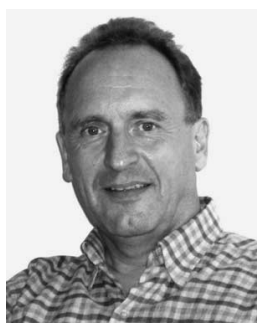

Erich Meier received the M.Sc. degree in geography and the Ph.D.(Hons.) degree in remote sensing from the University of Zurich, Zurich, Switzerland, in 1982 and 1989 , respectively.

From 1979 to 1983, he was a Research Assistant with the Department of Geography, University of Zurich. From 1983 to 2006, he was a Lecturer and Research Scientist with the Remote Sensing Laboratories (RSL), University of Zurich, where he is now a Research Section Head. Since 2000, he has been the Head of the SARLab, a research group within RSL. The main research interests of this group are the development of new focusing algorithms for SAR data from UHF to $\mathrm{mmW}$, interferometry, polarimetry, and MTI algorithms, as well as calibration and validation activities for spaceborne and airborne systems. He is responsible for the research strategies as well as the organization of the team. He is Consultant on behalf of several national and international organizations and private companies.

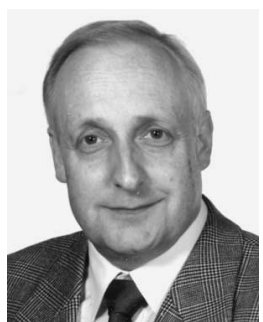

Daniel Nüesch (M'84) received the M.S. degree in cartography and the $\mathrm{Ph} . \mathrm{D}$. degree in remote sensing from the University of Zurich, Zurich, Switzerland, in 1972 and 1976, respectively.

From 1977 to 1978 and from 1980 to 1982 , he was a European Space Agency (ESA) Fellow at the Environmental Research Institute of Michigan, Ann Arbor, performing National Aeronautics and Space Administration contract work including Landsat-TM spectral band simulations, thermal infrared studies, and microwave projects, such as investigations of augmenting Landsat-MSS data with Seasat SAR imagery. Since 1982, he has worked as a Research Scientist with the Remote Sensing Laboratories, University of Zurich, and was involved in teaching and leading a large research group dedicated in particular to geometric and radiometric SAR calibration. As Scientific Division Head and Professor at the University of Zurich, he supervised $12 \mathrm{Ph} . \mathrm{D}$. and numerous postdoctoral students. As Principal Investigator, Coinvestigator, and Head of ESA's Expert Support Laboratory, he was involved in several ESA investigations for ERS-1/2 and Envisat. Since 1980, he has served as a Swiss delegate to ESA in the Data Operations Scientific and Technical Advisory Group and in the Earth Observation Programme Board (PB-EO).

Dr. Nüesch is a member of the Swiss Federal Space Affairs Commission (CFAS). 